passes, and increased rates of first pass success without increased risk of intracranial hemorrhage. These results are in contrast to the increased hemorrhage rates reported in $\mathrm{AF}$ associated stroke treated with supportive care and or intravenous thrombolysis. Bridging therapy in AF patients undergoing thrombectomy independently increased the odds of intracranial hemorrhage and did not improve functional outcomes. Together, these results suggest that AF associated stroke has a differential response to intravenous thrombolysis and mechanical thrombectomy. Randomized trials are warranted to determine whether patients with $\mathrm{AF}$ associated stroke may benefit by deferring bridging therapy at thrombectomy-capable centers.

Disclosures F. Akbik: None. A. Alawieh: None. C. Cawley: None. B. Howard: None. F. Tong: None. F. Nahab: None. F. Nahab: None. H. Saad: None. L. Dimisko: None. O. Samuels: None. G. Pradilla: None. I. Maier: None. W. Feng: None. R. Chalhoub: None. N. Goyal: None. R. Starke: None. A. Rai: None. K. Fargen: None. M. Psychogios: None. P. Jabbour: None. R. De Leacy: None. S. Keyrouz: None. T. Dumont: None. P. Kan: None. J. Liman: None. A. Arthur: None. D. Mccarthy: None. V. Saini: None. S. Wolfe: None. J. Mocco: None.

\section{LB-003 ISCHEMIC STROKE ASSOCIATED WITH COVID-19 AND RACIAL OUTCOME DISPARITY IN NORTH AMERICA}

${ }^{1} \mathrm{~A}$ Dmytriw ${ }^{*},{ }^{1} \mathrm{~K}$ Phan, ${ }^{2} \mathrm{C}$ Schirmer, ${ }^{3} \mathrm{~F}$ Settecase, ${ }^{3} \mathrm{M}$ Heran, ${ }^{4} \mathrm{~A}$ Efendizade, ${ }^{5} \mathrm{~A}$ Kuhn, ${ }^{5} \mathrm{~A}$ Puri, ${ }^{6} \mathrm{~B}$ Menon, ${ }^{7} \mathrm{M}$ Dibas, ${ }^{8} \mathrm{~S}$ Sivakumar, ${ }^{9} \mathrm{~A}$ Mowla, ${ }^{10} \mathrm{~L}$ Leung, ${ }^{10} \mathrm{~A}$ Malek, ${ }^{11} \mathrm{~B}$ Voetsch, ${ }^{11} \mathrm{~S}$ Segal, ${ }^{11} \mathrm{~A}$ Wakhloo, ${ }^{12} \mathrm{H}$ Wu, ${ }^{13} \mathrm{~A}$ Xavier, ${ }^{12} \mathrm{~A}$ Tiwari. ${ }^{1}$ Harvard University, Boston, MA, Canada; ${ }^{2}$ Geisinger, Wilkes-Barre, PA; ${ }^{3}$ Vancouver General Hospital, Vancouver, $B C$, Canada; ${ }^{4}$ SUNY Downstate Hospital, New York, NY; ${ }^{5}$ UMass Memorial Medical Center, Worcester, MA; ${ }^{6}$ Calgary Cumming School of Medicine, Calgary, AB, Canada; ${ }^{7}$ Harvard University, Boston, MA; ${ }^{8}$ Prisma Health, Greenville, SC; ${ }^{9}$ Keck School of Medicine USC, Los Angeles, CA; ${ }^{10}$ Tufts University Hospital, Boston, MA; ${ }^{11}$ Lahey Hospital Health Center, Burlington, MA; ${ }^{12}$ Brookdale University Hospital, Brooklyn, NY; ${ }^{13}$ Sinai Grace Hospital, Detroit, MI

\subsection{6/neurintsurg-2020-SNIS.275}

Introduction There has been limited evidence on the influence of racial background in stroke outcomes in COVID-19. Underlying biological, genetic, or epigenetic characteristics may predispose to health differences and outcomes. Social determinants of health, access and geographical differences pertaining both to population density and other location-based factors may also be important.

Methods We report 69 cases of acute stroke in patients positive for SARS-CoV-2, including 27 of African American background and 42 of other racial backgrounds, including Caucasian, Hispanic, and Asian. All patients presented to 14 major hospitals in the United States and Canada, from March 14-April 14, 2020. All patients had nasopharyngeal swab samples that were positive for SARS-CoV-2 on qualitative RT-PCR assays. We present a dichotomized analysis of ischemic stroke outcomes between patients of African American background as reported on hospital intake questionnaire versus all other backgrounds.

Results Comparison between Caucasian, Hispanic and Asian backgrounds did not show disparities in stroke outcomes. We found no significant difference in age (64.4 versus 62.9 years) or the proportion of females $(51.9 \%$ versus $38.1 \%)$ (table 1). Diabetes mellitus was present significantly less in African American cases versus others (37\% vs. 66.7\%). No significant difference between groups was found regarding other comorbidities including smoking, atrial fibrillation, prior anticoagulation, coronary artery disease, congestive heart failure, hypertension, hyperlipidemia, cerebrovascular accident, peripheral vascular disease, or chronic kidney disease. With respect to presenting SARS-CoV-2 symptoms, we found no difference in exposure history, asymptomatic cases, fever, cough, dyspnea, nausea or vomiting, chills, malaise, or lethargy. The African American cohort had a similar mean NIHSS score of 16.3 compared with 14.9 in other races $(p=0.63)$. The door-to-CT time was also similar (23 versus 19 minutes). The proportion of patients presenting with a large vessel occlusion was not significantly different $(40.7 \%$ versus $47 \%$ ). We noted $14.8 \%$ of African American cases received intravenous tPA compared to $31 \%$ in other races, but this was not significantly different in this sample. The proportion of thrombectomy cases mirrored this $(14.8 \%$ versus $31 \%$ ). Laboratory findings were not significantly different between African Americans and all others. Regarding stroke functional outcomes, there was no difference between African Americans and other races in terms of discharge mRS $(\mathrm{p}=0.27)$. For mRS $0-2$, there was no significant difference noted $(14.8 \%$ versus $16.7 \%)$. Symptomatic intracranial hemorrhage (sICH) was significantly higher for African Americans $(11.1 \%$ versus $3 \%, p<0.001)$. Mortality was significantly higher in African Americans compared to other races $151.9 \%$ vs. $28.6 \%, \mathrm{p}=0.03)$.

Discussion Our preliminary data suggest that there may be a mortality difference amongst stroke patients of African American background afflicted with COVID-19. The reasons for increased mortality in African Americans with COVID-19-associated stroke are unknown. Racial disparities in case counts and outcomes during the COVID-19 pandemic have been highlighted, particularly regarding African American communities. In addition, the finding in this study that mortality rate of COVID-19 positive stroke patients is greater than that previously reported in either COVID-19 respiratory infection alone or acute ischemic stroke alone, suggests an interaction that also warrants further study.

Disclosures A. Dmytriw: None. K. Phan: None. C. Schirmer: None. F. Settecase: None. M. Heran: None. A. Efendizade: None. A. Kuhn: None. A. Puri: None. B. Menon: None. M. Dibas: None. S. Sivakumar: None. A. Mowla: None. L. Leung: None. A. Malek: None. B. Voetsch: None. S. Segal: None. A. Wakhloo: None. H. Wu: None. A. Xavier: None. A. Tiwari: None.

\section{LB-004 SEX DIFFERENCES IN ACUTE STROKE THROMBECTOMY MANAGEMENT AND OUTCOMES IN THE UNITED STATES: EVIDENCE FROM THE NVQI-QOD REGISTRY}

${ }^{1} \mathrm{~V}$ Thirunavu ${ }^{*},{ }^{2} \mathrm{R}$ Abdalla, ${ }^{2} \mathrm{D}$ Cantrell, ${ }^{3} \mathrm{M}$ Hurley, ${ }^{3} \mathrm{~A}$ Shaibani, ${ }^{1} \mathrm{~B}$ Jahromi, ${ }^{1} \mathrm{M}$ Potts, ${ }^{4} S$ Ansari, ${ }^{5} \mathrm{O}$ NVQI-QOD Registry. ${ }^{1}$ Department of Neurological Surgery, Northwestern University Feinberg School of Medicine, Chicago, $L_{\text {; }}{ }^{2}$ Department of Radiology, Northwestern University Feinberg School of Medicine, Chicago, IL; ${ }^{3}$ Department of Radiology, Department of Neurological Surgery, Northwestern University Feinberg School of Medicine, Chicago, IL; ${ }^{4}$ Department of Radiology, Department of Neurological Surgery, Department of Neurology, Northwestern University Feinberg School of Medicine, Chicago, IL; ${ }^{5}$ SNIS, M2S, NPA, AANS, Fairfax, VA

\subsection{6/neurintsurg-2020-SNIS.276}

Introduction Sex differences in outcomes after acute ischemic stroke have been documented in the literature. However, few 
studies have assessed sex differences in outcomes after endovascular stroke thrombectomy of large vessel occlusion (LVO), and none have explored the relationship in a robust realworld registry.

Methods Data from the NeuroVascular Quality InitiativeQuality Outcomes Database (NVQI-QOD) registry was analyzed and compared for sex differences in stroke thrombectomy outcomes in 3261 patients from 23 US centers (17 states) between January 2015 to March 2020. Welch's twosided t-tests, Chi-square tests, and multivariate regression models were used to assess sex differences for 11 outcome variables: final thrombolysis in cerebral infarction (TICI) grade $(\mathrm{n}=3182), 24$ hour NIH stroke score (NIHSS) $(n=2842)$, post-procedure length of stay $(n=3257)$, ICU days $(\mathrm{n}=2787)$, in-hospital mortality $(\mathrm{n}=3259)$, discharge status $(n=3252)$, discharge NIHSS $(n=2426)$, discharge modified Rankin score (mRS) $(n=996)$, days to follow-up $(n=1784)$, 90 day readmission rate $(n=416)$, and 90 day mRS $(n=1184)$. Models controlled for demographics, comorbidities, intravenous tPA thrombolysis, and pre-stroke functional measures.

Results Among the 3261 patients that underwent stroke thrombectomy, $49.4 \%$ were female $(n=1622)$ and $50.6 \%$ were male $(n=1639)$. T-tests and Chi-square tests revealed significant differences between females and males for postprocedure length of stay (8.1 versus 9.1; $\mathrm{p}=0.019), 24$ hour NIHSS (11.1 versus $10.0 ; \mathrm{p}=0.001)$, discharge status $(30.8 \%$ home versus $34.5 \%$ home; $\mathrm{p}<0.001)$, days to follow-up (110 versus $126 ; \mathrm{p}=0.008)$, and in-hospital mortality $(25.3 \%$ versus $19.9 \% ; \mathrm{p}<0.001)$. There were no significant sex differences for discharge mRS without mortality (Female: $27.0 \%$ favorable outcome, Male: $26.7 \%$; $\mathrm{p}=0.95$ ) or for 90 day mRS without mortality (Female: $52.8 \%$ favorable outcome, Male: $49.5 \%$; $\mathrm{p}=0.60)$. Multivariate regression models revealed that females had significantly higher 24 hour NIHSS $(p=0.004)$, fewer days to follow-up $(p=0.033)$, and higher rate of in-hospital mortality $(p=0.036)$. The interaction term of African American $x$ Male and Hispanic x Male were significant for post-procedure length of stay and ICU days, respectively $(p=0.026$ and $\mathrm{p}=0.022$ ), indicating a differential effect of race on the outcome variable within males.

Conclusion Evidence from the NVQI-QOD registry suggests that there are differences between males and females in outcomes after acute stroke thrombectomy, with females showing consistently worse outcomes for short-term functional status, morbidity, and early mortality. Finally, there appears to be a differential effect of race on certain outcome variables within males.

Disclosures V. Thirunavu: None. R. Abdalla: None. D. Cantrell: None. M. Hurley: None. A. Shaibani: None. B. Jahromi: None. M. Potts: None. S. Ansari: None. O. NVQIQOD Registry: None.

\section{LB-005 UNRUPTURED ARTERIOVENOUS MALFORMATION INTERVENTION RATE IS INVERSELY CORRELATED WITH RUPTURED AVM DISCHARGE INCIDENCE}

'D McCarthy*, E Luther' ${ }^{2}$, B Gross ${ }^{1}$, R Starke ${ }^{3}$. 'University of Pittsburgh Medical Center, Pittsburgh, PA; ${ }^{2}$ University of Miami, Miami, FL; ${ }^{3}$ University of Miami Miller School of Medicine, Miami, FL
Introduction The 2013 ARUBA trial concluded that medical management alone is superior to medical management with interventional therapy for patients with unruptured brain arteriovenous malformations.

Objective To analyze AVM treatment and epidemiologic response to the ARUBA trial.

Methods National Inpatient Sample was reviewed from 2003 to 2017, ruptured and unruptured AVMs were identified (rAVM, uAVM). Periods before and after ARUBA defined as 2010-2013 and 2014-2017. Multivariable logistic regression identified correlates to intervention likelihood. Yearly intervention rate (YIR, \#interventions/\#AVM discharges) and yearly rAVM incidence (YRR, \#rAVM/\#AVM discharges) were calculated. Segmented regression (SR) identified significant changepoints and fitted segmented linear models for YIR and YRR. Average annual percent change (AAPC) was estimated as the weighted sum of segmented model slopes. Pearson coefficients (PC) examined correlation between YIR and YRR. RStudio performed statistics.

Results 90,296 AVM discharges were identified from 20102017. AVM discharges were seen at smaller, non-academic hospitals following ARUBA $(\mathrm{p}<0.0001)$. SR identified a significant change in YIR during 2014. Figure 2 shows a decrease in interventions for uAVMs $(28.1 \%$ vs. $22.3 \%$, $\mathrm{p}<0.0001$ ) and no change in rAVM interventions following ARUBA $(p=0.22)$. uAVM discharges following ARUBA had a less likelihood of intervention than those before (OR 0.857 , $\mathrm{p}=0.02$ ); however, intervention likelihood for uAVMs with associated aneurysms were unaffected by ARUBA. Since we unexpectedly observed a higher rAVM discharge incidence following ARUBA $(18.6 \%$ vs $14.7 \%, \mathrm{p}<0.0001)$, the analysis was expanded to 2003 (154,297 discharges). Overall, YRRs have increased (AAPC +0.49\%, $\mathrm{p}=0.0001$ ) and YIRs have decreased (AAPC -1.17\%, $p=0.0001$; figure $1 \mathrm{~A}$ ). The

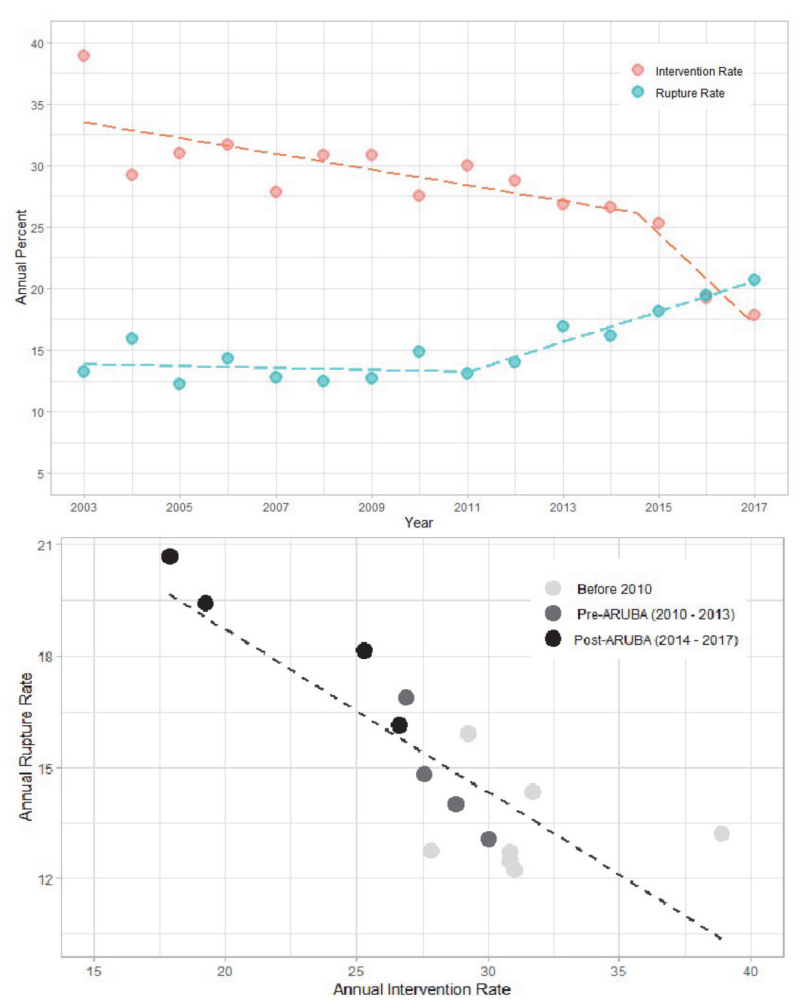

Abstract LB-005 Figure 1 